\title{
DISJUNCTIVE FEATURE STRUCTURES AS HYPERGRAPHS
}

\section{JEAN VIERONIS}

Groupe Représentation et Traitement des Connaissances,

Centre National de la Recherche Scientifique,

31. Chemin Joseph Aiguier, 13402 Marseilic Cedex 09, France

and

Department of Computer Science, Vassar College

Poughkeepsic. New York 12601, U.S.A.

e-mail : veronis lavassar.edu

\begin{abstract}
In this paper, we present a new mathematical framework in which disjunctive feature stnuctures are defined as directed acyclic hypergraphs. Disjunction is defined in the feature structure domain, and not at the syntactic level in feature descriptions. This enables us to study propertics and specify operations in terms of properties of, or operations on, hypergraphs rather than in syntactic terms. We illustrate the expressive power of this framework by defining a class of disjunctive feature structures with interesting properties (factored normal form or FNF), such as closure under factoring, unfactoring, unification, and generalization. Unification, in particular, has the intuitive appeal of preserving as much as possible the particular factoring of the disjunctive feature structures to be unified. We also show that unification in the FNF class can be extremely efficient in practical applications.
\end{abstract}

\section{INTRODUCTION}

It has become common to make a distinction between a language for the description of feature structures and feature structures themselves, which are secn as directed acyclic graphs (dags) or automata (see, for instance, Kasper and Rounds, 1986). To avoid confusion, the terms of the representation language are often referred to as feature descriptions. Disjunction is a representation tool in the representation language, intended to describe sets of feature structures. In this framework, there are no disjunctive featurc structures, but only disjunctive feature descriptions.

This framework has enabled researchers to explore the computational complexity of unification. However, it has some drawbacks. First, properties have to be stated (and proofs carried out) at the syntactic level. This implies using a complicated calculus based on formula equivalence rules, rather than using graph-theorctical properties. In addition, unification is not well-defined with respects to disjunction. There is reference in the literature to the "unification of disjunctive feature descriptions", but, formally, we should instead speak of the unification of the sets of feature structures the descriptions represent.

For example, unifying the sets of feature structures represented by the disjunctive fcature descriptions in Fig. 1 yields a set of four (non-disjunctive) feature structures, which can be described by scveral equally legitimate formulae: A factored, B factored, disjunctive normal form (DNF), etc. Depending on the algorithm that is used, the description of the result will be one or the other of these formulae. Some algorithms require expansion to DNF and will therefore produce a DNF representation as a result, but other algorithms may produce different representations.

There is an important body of research concerned with the development of algorithms that avoid the expensive expansion to DNF (c.g., Kasper, 1987). These algorithms typically produce descriptions of the unification, in which some of the disjunctions in the original descriptions arc retained. However, these descriptions are produced as a computational side-effect (potentially different depending on the algorithm) rather than as a result of the application of a formal definition.

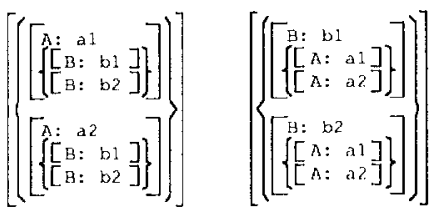

Fig. 1. Different descriptions for the same set of feature structures

In this paper, we first consider disjunctive feature structures as objects in themselves, defined in terms of directed acyclic hypergraphs. This enables us to build a mathematical framework based on graph theory in order to study the properties of disjunctive feature structures and specify operations (such as unification) in algebraic rather that syntactic terms. It also enables the specification of algorithms in terms of graph manipulations, and suggests a data structurc for implementation.

We then illustrate the expressive power of this framcwork by defining a class of disjunctive feature structures with interesting properties (factored normal form or $F N F$ ), such as closure under factoring, unfactoring, unification, and generalization. These operations (and the relation of subsumption) are defined in terms of operations on (or relations among) hypergraphs. Unification, in particular, has the intuitive appeal to preserve as much as possible the particular factoring of the disjunctive feature structures to be unificd. We also show that unification in the FNF class can be extremely efficient in practical applications.

For lack of space, proofs will be omitted or only suggested. 


\section{BASIC FRAMEWORK}

\subsection{Disjunctive feature stuctures as hypergraplis}

(Disjunctive) feature structures will be defined as directed acyclic hypergraphs. In a hypergraph (see Berge, 1970), arcs (hyperarcs) connect sets of nodes instead of pairs of nodes, as in usual graphs. We will consider hyperares as directed from their first node to all other nodes. More precisely, each hyperare will be an ordered pair consisting of an input node $n_{i p}$, and a (noncmpty) set of output nodes $n_{i_{j}}, \ldots, n_{i_{k}}$. We will say that $\left(n_{i_{0}},\left\{n_{i_{1}}, \ldots, n_{i_{k}}\right\}\right)$ is a $k$-arc from $n_{i_{0}}$ to $n_{i_{1}}, \ldots, n_{i_{k}}$, that $n_{i_{0}}$ is an immediate predecessor of $n_{i_{1}}, \ldots, n_{i_{k}}$, and that $n_{i}, \ldots, n_{i_{k}}$ are immediate successors of $n_{i_{0}}$.

$A$ path ${ }^{1}$ in a hypergraph is a sequence of nodes $n_{i}, \ldots, n_{i_{p}}$ such that for $j=1, \ldots, p-1, n_{i j}$ is an immediate predecessor of $n_{i j+1}$. If there exists a path from a node $n_{i}$ to a node $n_{j}$, we will write $n_{i} \Rightarrow n_{j}$. A hypergraph is acyclic if there is no node such that $n_{i} \Rightarrow$ $n_{i}$. A hypergraph has a root $n_{0}$ if for each node $n_{i} \neq n_{0}$, $n_{0} \Rightarrow n_{i}$. The leaves of a hypergraph are those nodes with no successor. A path terminating with a leaf is a maximal path. Nodes with more than one immediate predecessor are called merging nodes.

Definition 2.1 Let $L$ be a set of labels and $A$ be a set of atomic values. A (disjunctive) feature structure on ( $\mathrm{L}$, $A)$ is a quadruple $F=\left(D, n_{0}, \lambda, \alpha\right)$, respecting the consistency conditions 2.1 below, where $D$ is a finite directed acyclic hypergr'aph with a root $n_{0}, \lambda$ is a partial function from the 1 -arcs of $D$ into $L$, and $\alpha$ is a partial function from the leaves of $D$ into $A$.

Foature structures which have isomorphic hypergraphs, whose corresponding leaves have the same valuc, and whose corresponding feature-arcs have the same labels, are isomorphic. We will consider such feature structures to be equal up to isomorphism.

Definition 2.2 Labeled 1-arcs are called feature-arcs. Non-labeled hyperarcs are called OR-arcs.

Note that OR-arcs are usually $k$-arcs with $k>1$, but (non-labeled) 1-arcs can be $O R$-arcs as a special case. We will use a graphic representation for disjunctive feature structures in which OR-arcs are represented as $k$ lines connected together ${ }^{2}$ (see Fig. 2).

Definition 2.3 The extended label of a given path is the concatenation of all labels along that path. We will use the notation $l_{1}: l_{2}: \ldots l_{n}$ to represent extended labels. A maximal extended label from a node is an extended label for a maximal path from that node.

\footnotetext{
${ }^{1}$ We use this term in the sense usual in graph thecory. It should not be confused with the term path used in many fenture structure studies, which is a string of labels, and for which we will introduce the term exiended label later in the paper.

${ }^{2}$ In some work involving $A N D / O R$ graphs, this convention is used for AND-ancs. This should not create further confusion.
}

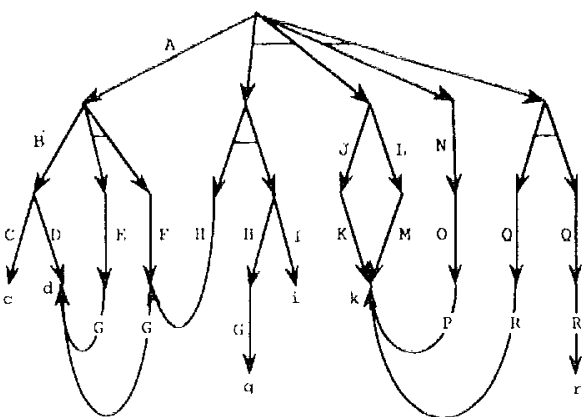

Fig. 2. Graplic representation

Conditions 2.1 Disjunctive feature structures must verify the following consistency conditions:

(C1) No output noxde of an ok-are is a leaf;

$\left(C_{2}\right)$ Output noxles of OR-arcs are not merging nodes;

(C.3) All feature-ares from the same node lave different latxels;

(C4) No maximal extended label from a given node is a prefix of a non-inaximal extended label obtained by following a different hyperanc from the same node.

$\mathrm{C}_{1}$ and $\mathrm{C}_{2}$ constrain $\mathrm{OR}$-arcs to represent only disjunctions. $C_{3}$ and $C_{4}$ are extensions of the determinisin that is usually imposed on dags (no outgoing arcs with the same label from any given node).

Definition $2.4 \mathrm{~A}$ dag feature structure is a fcature structure with no OK-arc.

Definition 2.5 A projection of a feature structure $x$ is a hypergraph obtained by removing all but one output node of all (OR-arcs of $x$.

Theretore, a projection has only 1 -arcs.

Definition 2.6 $\wedge$ dag feature structure $y$ is a $d a g$. projection of a feature structure $x$ if there exist some projection $y^{\prime}$ of $x$ and a function $h$ mapping nodes of $y^{\prime}$ into nodes of $y$ such that:

(1) the root of $y^{\prime}$ is mapped to the root of $y$;

(2) if $\left(n_{i_{0}},\left\{n_{i_{1}}\right\}\right)$ is a feature-arc of $y^{\prime}$, then $\left(h\left(n_{i_{0}}\right),\left\{h\left(n_{i_{1}}\right)\right\}\right)$ is a feature-arc of $y$ with the sime label;

(3) if $\left(n_{i_{0}},\left(n_{i_{1}}\right\}\right)$ is a $1-\mathrm{OR}-\operatorname{arc}$ of $y^{\prime}$, then $h\left(n_{i_{0}}\right)=$ $h\left(n_{i}\right)$;

(4) the value associated with a node $n_{i}$ in $y^{\prime}$ is the same as the value associated with $h\left(n_{i}\right)$ in $y$, or both have no valuc;

(5) each feature arc in $y$ is the image of at least one feature arc in $y^{\prime}$.

In other terms, a dag-projection is obtained from a projection by merging the input and output nodes of each 1-OK-arc, and merging paths with common prefixes to ensure determinism.

Definition 2.7 A sub-feature structure rooted at a node $n_{i}$ is a quadruple composed of a sub-hypergraph rooted at that node, the root $n_{i}$, together with the 
restrictions of the label and value functions to this subhypergraph. The AND-part of a node is the sub-feature structure rooted at that node, starting with only the feature-arcs from that node. The OR-parts of a node are the different sub-feature structures rooted at that node, starting with each of the OR-arcs. The disjuncts of an OR-arc are the sub-feature structures rooted at each of the output nodes of that $O R$-arc. If a node has only one OR-arc, we will call its disjuncts the disjuncts of the node.

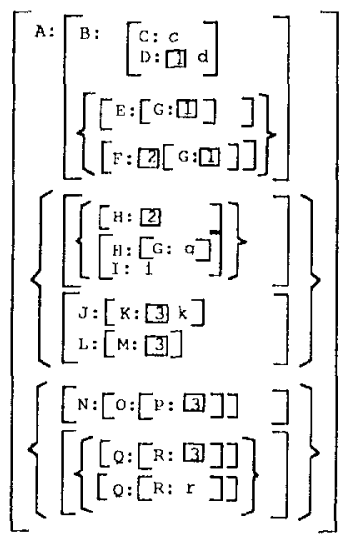

Fig. 3. Description of the feature structure in Fig. 2.

\subsection{Representation language}

Definition 2.8 The representation language for (disjunctive) feature structures described above is defined by the following grammar:

$$
\begin{array}{ll}
\mathrm{F} & \rightarrow[\mathrm{T}, \ldots, \mathrm{T}] \\
\mathrm{T} & \rightarrow l: \mathrm{IV} \\
\mathrm{T} & \rightarrow[\mathrm{F}, \ldots, \mathrm{F}] \\
\mathrm{I} & \rightarrow i \mid \varepsilon \\
\mathrm{V} & \rightarrow \mathrm{F}|a| \varepsilon
\end{array}
$$

where $F$ is the axiom, $\varepsilon$ is the empty string, $l$ belongs to the set of labels $\mathrm{L}, a$ belongs to the set of atomic values $A$, and $i$ belongs to a set I of identifiers (we use the symbols 1 , 国, etc.), disjoint from $L$. A formula $\Phi$ of that language is called a (disjunctive) feature description.

The mapping between feature structures and feature descriptions is straightforward (Fig. 3). Translating between feature descriptions and feature structures and checking that a description is valid (that is, corresponds to a valid feature structure) is computationally trivial, and does not rely on the (potentially expensive) application of equivalence rules as in Kasper and Rounds (1986).

\section{A TYPOLOGY OF NORMAL FORMS}

In this section, we will first define the disjunctive normal form (DNF) in terms of hypergraphs. We will then define a family of increasingly restricted normal forms, the most restricted of which is the DNF. One of them, the factored normal form (FNF) enables a clear definition of the "format" of a feature structure. It also imposes a strict hierarchical view of the data, and is exactly the class of feature structures that are reachable from the DNF through sequences of factoring operations. We believe that the FNF class is of great linguistic interest, since it is clear that disjunction is often used to reflect hicrarchical organization, factoring, etc., and thus is more than just a space-saving device. In the sections that follow, factoring operations in the FNF class will be defined formally, along with appropriate extentions to the notions of subsumption and unification.

\subsection{Disjunctive Normal Form}

Definition $3.1 \mathrm{~A}$ (disjunctive) feature structure is said to be in disjunctive normal form (DNF) if:

(1) the root has only one OR-part, and no AND-part;

(2) each disjunct is a dag feature structure;

(3) all the disjuncts are disjoint and different (nonisomorphic).

Note that the disjunctive normal form is defined for feature structures themselves, not for their descriptions.

Definition 3.2 The disjunctive normal form of a given feature structure $x$, noted $\operatorname{DNF}(x)$, is a DNF feature structure, in which the set of disjuncts $D_{i}$ is equal to the set of dag-projections of $x$.

Definition 3.3 Two feature structures $x$ and $y$ are $D N F$-equivalent if $\operatorname{DNF}(x)=\operatorname{DNF}(y)$. We will note $x \equiv_{d n s} y$.

\subsection{Typology of normal forms}

We can define several interesting restrictions on feature structures, which in turn define a typology of increasingly restricted normal forms.

Condition 3.1 Dag-projections obtained by different sclections of output nodes of $O R$-arcs are different.

Condition 3.2 Each node has at most one OR-part.

Condition 3.3 The AND-part of each node is a dag.

Definition 3.4 When combined, the three conditions above define several normal forms:

(1) 3.1: non-redundant normal form (NRNF);

(2) 3.1 and 3.2: hierarchical normal form (HNF);

(3) 3.1 and 3.3: AND-normal form (ANF);

(4) 3.1, 3.2 and 3.3: layered normal form (LNF).

Definition 3.5 In an ANF feature structure $x$, the AND-part of a node $n_{i}$ is a maximal AND-part of $x$ if $n_{i}$ is the output node of no feature arc.

Definition 3.6 The layers of a LNF feature structure are defined recursively as follows:

(I) Layer 0 is the AND-part of the root; 
(2) Layer $n+1$ is set of (maximal) AND-parts of all the output nodes of $\mathrm{OR}$-arcs originating in layer $n$.

Let us now tum back to formats.

Definition 3.7 The format of a dag feature structure is the set of maximal extended labels starting at its root. The format of a layer is the union of formats of all the maximal AND-parts in that laycr.

Definition 3.8 A LNF feature structure is said to be in factored normal form (FNF) if the following properties hold:

(1) the formats of all layers are disjoint;

(2) paths originating in two distinct maximal AND-parts of a layer $n$ can merge only in a node belonging to an AND-part in a layer $n^{\prime}$ such that $n^{\prime}<n$.

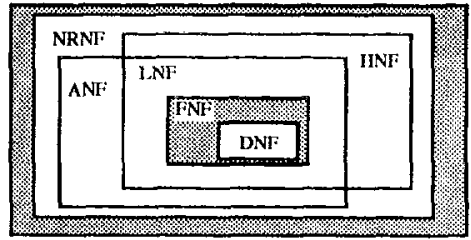

Fig. 3. A typology of normal forms.

Fig. 3 shows the typology of normal forms. Notc that the DNF is obviously in FNF.

In the rest of the paper, we will study only the properties of FNF, in which formats are homogeneous.

Definition 3.9 The formal of a FNF featurc structurc, noted $f(x)$, is the sequence $\left\langle s_{0}, \ldots, s_{n}\right\rangle$ of the formats of each of its layers, in increasing order starting with the root.

Definition 3.10 We will call sets of extended labels dag-formats, and sequences $\left\langle s_{0}, \ldots, s_{n}\right\rangle$ of dagformats with all $s_{i}$ disjoint, fs-formats.

Proposition 3.2 If two FNF feature structures have the same DNF and the same format, they are equal.

\section{RESTRUCTURING OPERATORS}

\subsection{Factor and unfactor}

Let us give first a few auxiliary definitions.

Definition 4.1 Let $x$ be a dag feature structure, and $s$ a dag-format. The spanning of $x$ according to $s$, noted $\operatorname{span}_{s}(x)$, is the greatest sub-dag of $x$ such that of all the paths in $\operatorname{span}_{s}(x)$ have their extended labels in $s$.

Note that $f\left(\operatorname{span}_{s}(x)\right) \subseteq s$.

Definition 4.2 A dag feature structure $F$ is a common factor of a feature structure $x$ if the AND-part of all the disjuncts at the top level of $x$ contain $F$. A dag format $s$ is said to span a common factor of $x$ if the spanning of the AND-part of all the disjuncts at the top level of $x$ according to $s$ is a common factor.

Let us now define the factoring and unfactoring operations. Informally, the factor operator extracts a factor common to all the top-level disjuncts, and raises it to the root level.

Definition 4.3 Let $x$ be a FNF feature structure such that $f(x)=\left\langle s_{0}, s_{1}, s_{2}, \ldots, s_{n}\right\rangle$ and $s$ a dag-format. If $s$ spans a common factor $F$, the factoring of $x$ according to $s$, noted $\phi_{s}(x)$, is the FNF feature structure DNFequivalent to $x$ with format $\left\langle s_{0} \cup s^{\prime}, s_{1}-s^{\prime}, s_{2}, \ldots, s_{n}\right\rangle$ where $s^{\prime}=f(F)$.

Definition 4.4 Let $x$ be a FNF feature structure with an AND-part $A$, such that $f(x)=\left\langle s_{0}, s_{1}, s_{2}, \ldots, s_{n}\right\rangle$, and $s$ be a dag-format. If $F=\operatorname{span}_{s}(x)$, the unfactoring of $x$ according to $s$, noted $\vec{\phi}_{s}(x)$, is the FNF feature structure that is DNF-equivalent to $x$ with the format $\left\langle s_{0}-s^{\prime}, s_{l} \cup s^{\prime}, s_{2}, \ldots, s_{n}\right\rangle$, where $s^{\prime}=f(F)$.

Example. Sec Fig. 4

Proposition $4.1 \phi_{s}\left(\bar{\phi}_{s}(x)\right)=\bar{\phi}_{s}\left(\phi_{s}(x)\right)=x$

Proposition $\mathbf{4 . 2}$

(1) $\phi_{s}\left(\phi_{s}(x)\right)=\phi_{s^{\prime}}\left(\phi_{s}(x)\right)=\phi_{s u s}(x)$

(2) $\bar{\phi}_{s}\left(\bar{\phi}_{s^{\prime}}(x)\right)=\bar{\phi}_{s}\left(\bar{\phi}_{s}(x)\right)=\bar{\phi}_{s \cup s^{\prime}}(x)$

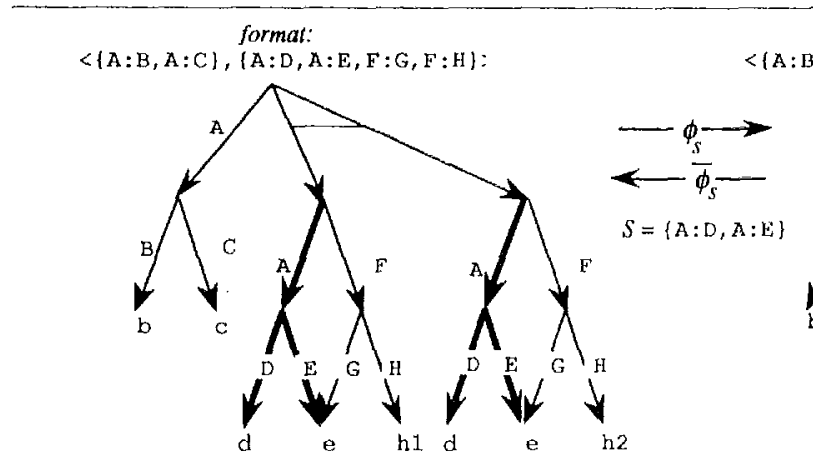

Fig. 4. Factoring and unfactoring

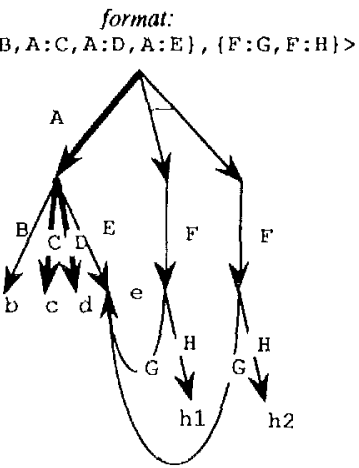




\subsection{Group and ungroup}

The factor operator requires that there is a common factor. In many cases there is no common factor; however, it is possible to define a group operator that first splits feature structures into groups of disjuncts that have common factors with respect to a given format, and then factors them.

Definition 4.5 Let $x$ be a FNF feature structure such that $f(x)=\left\langle\phi_{1}, s_{1}, s_{2}, \ldots, s_{n}\right\rangle, A_{1}, \ldots, A_{n}$ be the AND-parts of the top-level disjuncts of $x$ and $s$ be a dag-format. The grouping of $x$ according to $s$, noted $\gamma_{s}(x)$, is the FNF feature structure DNF-equivalent to $x$ with format $\left.<\emptyset, s^{\prime}, s_{1}-s^{\prime}, s_{2}, \ldots, s_{n}\right\rangle$ where $s^{\prime}=$ $\bigcup_{i} f\left(\operatorname{span}_{s}\left(A_{j}\right)\right)$.

Definition 4.6 Let $x$ be a FNF featurc structure such that $f(x)=\left\langle\emptyset, s_{1}, s_{2}, \ldots, s_{n}\right\rangle, A_{1}, \ldots, A_{n}$ be the AND-parts of the top-level disjuncts of $x, s$ be a dagformat, and $s^{\prime}=\bigcup_{i} f\left(\operatorname{span}_{s}\left(A_{i}\right)\right)$. We will note $\bar{\gamma}_{s}(x)$ the ungrouping of $x$ according to $s$ :

(1) if $s^{\prime}=s_{1}$ then $\vec{\gamma}_{s}(x)$ is the FNF feature structure DNF-equivalent to $x$ with format $\left\langle\phi, s_{1} \cup s_{2}, s_{3}, \ldots, s_{n}\right\rangle$;

(2) if $s^{\prime} \neq s_{1}$ then $\gamma_{s}(x)$ is the FNF feature structure DNF-equivalent to $x$ with format $\left\langle\emptyset, s_{1}-s^{\prime}, s^{\prime}\right.$ $U s_{2}, s_{3}, \ldots, s_{n}>$.

Example. See Fig. 5.

Proposition $4.3 \gamma_{s}\left(\bar{\gamma}_{s}(x)\right)=\bar{\gamma}_{s}\left(\gamma_{s}(x)\right)=x$

Proposition 4.4 The class of FNF feature structures is closed under factoring, unfactoring, grouping and ungrouping.

\subsection{Format operator}

Definition 4.7 Let $S$ be a fs-format $\left\langle s_{0}, s_{1}, \ldots, s_{n}\right\rangle$. The formatting of a DNF feature structure $x$ according to $S$, noted $v_{S}(x)$, is the result of the following sequence of operations:

$$
v_{S}(x)=\phi_{s_{0}}\left(\gamma_{s_{1} \cup s_{0}}\left(\gamma_{s_{2} \cup s_{1} \cup s_{0}}\left(\ldots\left(\gamma_{s_{n}} \cup \ldots \cup s_{0}(x)\right)\right)\right)\right.
$$

It is clear that $v_{S}(x)$ is in FNF, and is DNFequivalent to $x$.

Proposition 4.5 Any FNF feature structure $x$ can be reached from its DNF though a sequence of grouping and factoring operations. More precisely, if $x^{\prime}=$ $\mathrm{DNF}(x)$ then $x=v f(x)(x)$.

Definition 4.8 Let $S$ be a fs-format $\left\langle s_{0}, s_{1}, \ldots, s_{n}\right\rangle$. The unformatting of a FNF feature structure $x$ according to $S$, noted $\bar{v}_{S}(x)$, is the result of the following sequence of operations:

$\bar{v}_{S}(x)=\bar{\gamma}_{s_{n} \cup \ldots \cup s_{0}}\left(\ldots\left(\bar{\gamma}_{s_{2} \cup s_{1} \cup s_{0}}\left(\bar{\gamma}_{s_{1} \cup s_{0}}\left(\bar{\phi}_{s_{0}}(x)\right)\right)\right)\right.$

Proposition 4.6 Any FNF feature structure $x$ can be transformed into its DNF though a sequence of unfactoring and ungrouping operations. More preciscly, $\vec{v}_{f(x)}(x)=\mathrm{DNF}(x)$.

Proposition $4.7 v_{S}\left(\bar{v}_{S}(x)\right)=\ddot{v}_{S}\left(v_{S}(x)\right)=x$

\section{SUBSUMPTION, UNIFICATION AND GENERALIZATION}

As mentioned in the introduction, the format of the result of unification is not defined in the classical approach. Our goal will be to define unification on FNF disjunctive feature structures in such a way that the format of the result is unique and predictable. Intuitively, when feature descriptions have compatible formats (as in Fig. 6), it secms that unification should preserve it. On the other hand, when two feature descriptions have completely incompatible formats (as in Fig. 1), the resulting format should be in DNF. When formats are only partially compatible, a limited amount of unfactoring should be performed, and the compatible part should be preserved in the result. These considerations lead us to define compatibility of formats, and to extend the notions of subsumption, unification, and generalization to feature structure formats. We then define unification and gencralization on disjunctive feature structures in such a way that important properties

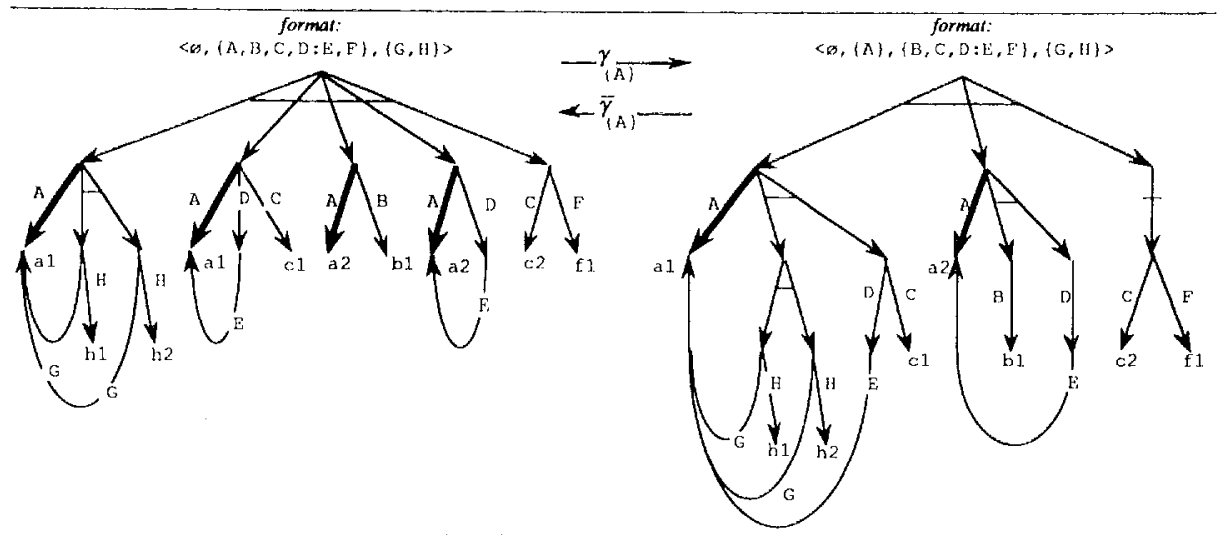

Fig. 5. Grouping and ungrouping 
hold. In particular, reduction to DNF, factoring, and grouping are homomorphisms with respect to unification (that is, $\operatorname{DNF}(x \sqcup y)=\operatorname{DNF}(x) \sqcup \operatorname{DNF}(y), \gamma_{s}(x \sqcup y)$ $=\gamma_{s}(x) \cup \gamma_{s}(y)$, etc. $)$.

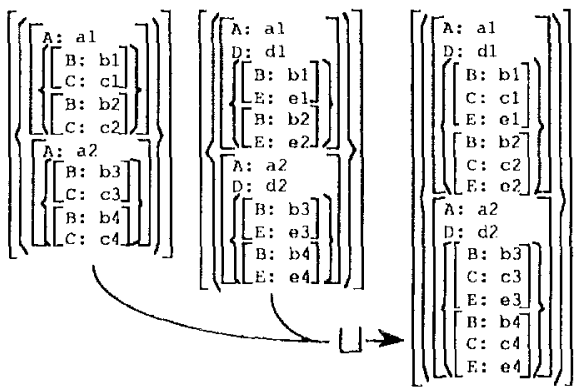

Fig. 6. Compatible formats

In what follows, we will call the classical subsumption, unification, generalization of dag feature structures dag-subsumption, dag-unification and daggeneralization (noted $\leq_{d a g}, \sqcup_{d a g}, \Pi_{\text {dag }}$, repectively). The classical subsumption, unification, gencralization of DNF feature structures will be called $d n f$-subsumption, dnf-unification and dnf-generalization (noted $\leq_{d n j}, \cup_{d n f}$, $\Pi_{\text {dny, }}$ repectively).

\subsection{Subsumption, unification, generalization of formats}

Definition 5.1 Let $s_{1}$ be a fs-format $<s_{1_{0}}$. $s_{1_{1}}, \ldots, s_{1_{n}}>$ and $S_{2}$ be a fs-format $\left\langle s_{2_{0}}, s_{2_{1}}, \ldots, s_{2_{p}}>\right.$. We will say that $S_{1}$ subsumes $S_{2}$ if each $p$ in $s_{1}$ belongs to some $s_{2}$ with $i \leq j$, for all $i$ in $\{1, n\}$. We will note $S_{1} \leq_{j m t} S_{2}$

Definition 5.2 Let $S_{1}$ and $S_{2}$ be two fs-formats. The unification of $S_{1}$ and $S_{2}$, noted $S_{1} \cup_{f m t} S_{2}$. is the greatest lower bound of $S_{1}$ and $S_{2}$ according to the format subsumption relation. The generalization of $S_{1}$ and $S_{2}$, noted $S_{1} \Pi_{\mathrm{fm}} S_{2}$, is the least upper bound of $S_{1}$ and $S_{2}$ according to the format subsumption relation.

It is easy to prove that these bounds exist. They can be built recursivcly. For example, let $S_{1}=\left\langle s_{1_{0}}\right.$, $\left.s_{1}, \ldots, s_{1_{n}}\right\rangle$ and $s_{2}=\left\langle s_{2_{0}}, s_{2}, \ldots, s_{2_{n}}\right\rangle$ (for the sake of simplicity, we will consider the shorter of $S_{1}$ and $S_{2}$ to be padded on the right with an appropriate number of $\emptyset$ 's in order to ensure the same length). $S=$ $s_{1} U_{f m t} s_{2}=\left\langle s_{0}, s_{1}, \ldots, s_{n}\right\rangle$ can be constructed recursively:

(1) $s_{\mathrm{n}}=s_{1_{\mathrm{n}}} \cup s_{2_{\mathrm{n}}}$

(2) $s_{i}=\left(s_{1_{i}} \cup s_{2_{i}}\right)-\bigcup_{j=1}^{n} s_{j}$ for all $i, 0 \leq i<n$.

Definition 5.3 Let $S_{1}$ be a fs-format $<s_{0}$, $\left.s_{1_{1}}, \ldots, s_{1_{n}}\right\rangle$ and $S_{2}$ a fs-format $\left\langle s_{2_{0}}, s_{2_{1}}, \ldots, s_{2_{p}}\right\rangle$. We will say that $S_{2}$ is a sub-format of $S_{1}$ if $s_{10}$ is included in $s_{2_{0}}$ for all $i$ in $\{1, n\}$. We will say that $S_{1}$ and $S_{2}$ are compatible if both $S_{1}$ and $S_{2}$ are sub-formats of the same format.
5.2 Subsumption, unification, generalization of disjunctive feature structures

Definition 5.4 We will say that a FNF feature structure $x$ subsumes a FNF feature structure $y$, and note $x \leq y$, if

$$
x \leq_{d n f} y
$$$$
\text { (2) } f(x) \leq_{f m t} f(y)
$$

Definition 5.5 Let $x$ and $y$ be two FNF featurc structures. The unification of $x$ and $y$, noted $x \downarrow y$, is the greatest lower bound of $x$ and $y$ according to the subsumption relation. The generalization of $S_{1}$ and $S_{2}$. noted $x \Pi y$, is the least upper bound of $x$ and $y$ according to the format subsumption relation.

The following proposition states that $x \sqcup y$ is dnfequivalent to the dnf-unification of the DNFs of $x$ and $y$, and the format of $x \cup y$ is the unification of the formats of $x$ and $y$ :

Proposition 5.1

(1) $\mathrm{DNF}(x \sqcup y)=\operatorname{DNF}(x) \sqcup_{d n f} \operatorname{DNF}(y)$
(2) $f(x \sqcup y)=f(x) \cup_{f m f} f(x)$

As a result, the unification of $x$ and $y$ can be computed by completely unformatting both $x$ and $y$, unifying them, and formatting the result according to the unification of their formats:

\section{Proposition 5.2 \\ $x \sqcup y=v_{f(x) \cup_{f m l} f(y)}\left(\bar{v}_{f(x)}(x) \sqcup_{d n f} \bar{v}_{f(y)}(y)\right)$}

(Dual proposition holds for generalization.)

Proposition 5.3 The class of FNF feature structures is closed under factoring, unfactoring, unification, and generalization.

This follows directly from the definitions.

\section{Proposition 5.4}

$$
\begin{aligned}
& \text { (1) } \quad \gamma_{s}(x \sqcup y)=\gamma_{s}(x) \sqcup \gamma_{s}(y) \\
& \text { (2) } \bar{\gamma}_{s}(x \sqcup y)=\bar{\gamma}_{s}(x) \sqcup \bar{\gamma}_{s}(y) \\
& \text { (3) } \phi_{s}(x \sqcup y)=\phi_{s}(x) \sqcup \phi_{s}(y) \\
& \text { (4) } \quad \bar{\phi}_{s}(x \| y)=\bar{\phi}_{s}(x) \sqcup \bar{\phi}_{s}(y)
\end{aligned}
$$

(Dual propositions hold for generalization.)

\subsection{Algorithm}

Proposition 5.2 does not imply that complete unfactoring and re-factoring is the most efficient computation of unification and generalization. Because of the properties given in proposition 5.4, unification can be carried out layer by layer, and only partial unfactoring is needed (algorithm 5.1). In the extreme case, when the formats of $x$ and $y$ are compatible, no unfactoring is needed, and the procedure match-formats does nothing. 
Algorithm 5.1 Unification of FNF feature structures

function unify $(x, y$ : feature-structure): feature-structure match-formats $(x, y)$

// Unify AND-parts

$z . A N D \leftarrow$ dag-unify (x.AND, y.AND)

if $z$.AND = failure then retum failure

// Unify OR-parts

$z . \mathrm{OR} \leftarrow$ unify-disjuncts $(x . \mathrm{OR}, y . \mathrm{OR})$

If $z . O R=$ failure then return failure else retum $z$

function unify-disjuncts $(x, y$ : feature-structure):

//assume $x . A N D$ and $y$. AND are emply

feature-structure

match-formats $(x, y)$

$k \leftarrow 0$

for each $x$. DISJ $_{j}$

for each $y$. DISJ $_{j}$

$t \leftarrow$ dag-unify $\left(x\right.$.DISI $_{j}$.AND, $y$.DISJ J $_{j}$.AND)

if $t \neq$ failure then

$u$ \& unify-disjuncts

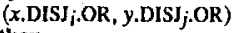

if $u \neq$ failure then

$k \leftarrow k+1$

z.DISJ $k$.AND $\leftarrow t$

$z$.DISJ $k$.OR $\leftarrow u$

if $k=0$ then return failure elsereturn $z$

We will consider the complexity of this algorithm in terms of the number of dag-unifications, which is the only costly operation $(\mathrm{O}(n \log (n))$, where $n$ is the total number of symbols in the two dag feature structures-see Aitt-Kaci, 1984). We will first consider the case where the formats are compatible. One dag-unification is performed in the unify function, but the bulk of the dagunifications are performed in the unify-disjuncts function. There are two nested loops, and the function is applied recursively through all the layers. Therefore, in the worst case, the algorithm requires $\mathrm{O}\left(d^{2}\right)$ dagunifications, whre $d$ is the total number of disjuncts.

When the formats are not compatible, some unfactoring and ungrouping has to be performed by the match-formats function in order to force the formats to match. The number of operations can be limited if the two formats are partially compatible, due to the properties of FNF. Complete unformatting will be necessary only in cases where the two formats are completely incompatible.

For example, if $f(x)=<\{A\},(B, C),[D, E\},[F]$, $\{G\},\{H]>$, and $f(y)=\langle[I\},(B, J\},\{D, F\},\{E, K\}$, $\{G\},\{L\}>$, the resulting format is $\langle\{A, I\},\{B, C, J\}$, [D\}, $(\mathrm{E}, \mathrm{F}, \mathrm{K}),[\mathrm{G}\},[\mathrm{H}, \mathrm{L}\}>$. The two first layers can be computed without unfactoring. Unfactoring is required for disjuncts at the next level, yielding the formats $<\{D\},\{E, F\},\{G\},\{H\}>$ and $<[D\}$, $\{E, F, K\},\{G],\{L\}>$, respectively. When this is accomplished the formats match, and the algorithm can resume with no more unfactoring.

It is clear that, in the worst case, when the algorithm requires the complete unformatting of the two feature structures, the total number of dag-unifications grows exponentially with the number of disjuncts. However, in most pratical cases, the algorithm is likely to perform better. We saw, in particular, that when the two feature structures have completely compatible formats, the complexity is only quadratic. There is obviously a range of possible behaviors between these two extremes.

It seems to us that in practical applications, disjunction is not random, but, instead, reflects some systematic linguistic properties. A high degree of compatibility among formats is therefore expected. It should also be noted that the algorithm can easily be modified so that only one feature structure is unfactored and re-formatted into a format that is compatible with the format of the other. This is especially useful in the common situation in which a small feature structure, containing a small number of disjuncts (e.g. a constituent at a given stage of parsing) is matched against a very large feature structure (c.g. a grammar). In this case, the time required for unformatting and reformatting the "small" feature structure is negligible, and the overall number of dag-unifications grows linearly with the number of disjuncts in the "large" feature structure.

\section{CONCLUSION}

In this paper, we present a new mathematical framework in which disjunctive feature structures are defined as directed acyclic hypergraphs. Disjunction is defined in the feature structure domain, and not at the syntactic level in feature descriptions. This enables us to study properties and specify operations (such as unification) and relations (such as subsumption) in terms of algebraic operations on (or relations among) hypergraphs rather than in syntactic terns. We illustrate the expressive power of this framework by defining a class of disjunctive feature structures with interesting properties (factored normal form, or FNF), such as closure under factoring, unfactoring, unification, and generalization. Unification, in particular, has the intuitive appeal of preserving as much as possible the particular factoring of the disjunctive feature structures to be unified. We also show that unification in the FNF class can be extremely efficient in practical applications.

Acknowledgments -. The present research has been partially funded by the GRECO-PRC Communication Homme-Machine of the French Ministery of Research and Technology and U.S.+French NSF/CNRS grant INT9016554 for collaborative research. The author would like to thank Nancy Ide for her valuable comments and help in the preparation of this paper.

\section{REFERENCES}

AlT-KACI, H. (1984) A New Model of Compulation Based on a Calculus of Type Subsumption. Ph. D. Thesis, Univ. of Pennsylvania.

BERGE, C. (1970). Graphes et Hypergraphes. Paris: Dunod. [translation: Graphs and Hypergraphs, Amsterdam : North-Holland, 1973]

KASPER, R. T. (1987). A unification method for disjunctive feature descriptions. Proc. 25 th Annual Meeting of the Association for Computational Linguistics. Stanford, California, 235-242.

KASPER, R., ROUNDS, W. C. (1986). A logical semantics for feature structures. Proc. 24th Annual Meeting of the Association for Computational Linguistics. New York, 257-266. 